\title{
Postpericardiotomy syndrome and its complications: prevalence, clinical significance and management
}

Taalaibek Z. Kudaiberdiev, MD, PhD, DSc.

Scientific Research Institute of Heart Surgery and Organ Transplantation, Bishkek, Kyrgyzstan

\begin{abstract}
:
In this review, we summarized current evidence on clinical significance, outcomes and management of postpericardiotomy syndrome and its complications developing after cardiac operations.
\end{abstract}

Key words: heart surgery, pericardium, postpericardiotomy syndrome, outcomes, management

(Heart-Vessels and Transplantation 2017: 1; 55-61. doi: 1024969/hvt.2017.10)

\section{Introduction}

Postpericardiotomy syndrome (PPS) occurs in about $15-30 \%$ of patients undergoing cardiac surgery $(1-6,7)$. It is defined in presence of 2 of 5 criteria: 1) fever occurring at least one week after surgery without underlying infection 2) pleuritic pain, 3) pericardial rub 4) pleural effusion and 5) pericardial effusion (8). Another research group, proposed more detailed definition of PPS, to minimize false-positive diagnosis providing details on the size of pleural and pericardial effusion and fever (7). In their definition, PPS is diagnosed in presence of 2 of the following criteria: pericardial rubbing, fever $>72$ hours postoperative, pleuritic chest pain, new significant pleural effusion on chest radiograph (above the highest level of the diaphragm), or significant pericardial effusion $(10 \mathrm{~mm})$. The ESC 2015 guidelines definition of PPS is under umbrella of post-cardiac injury syndrome, a group of inflammatory pericardial syndromes that include post-myocardial infarction Dressler pericarditis, PPS and post-cardiac trauma (9).
Postcardiac injury syndrome is diagnosed when at least 2 of 5 criteria met: 1) fever without alternative causes, 2) pericarditic of pleuritic chest pain, 3) pericardial or pleural effusion, 4) evidence of pericardial effusion and/or pericardial effusion with elevation of C-reactive protein. PPS can manifest as acute pericarditis as well (10). The main complications of PPS are development of tamponade and constrictive pericarditis (1-6). Tamponade complicates PPS in $6.4 \%$ and constrictive pericarditis in $2-3 \%$ of patients (11, 12).

We aimed to summarize current evidence on prevalence, clinical significance, outcomes and management of postpericardiotomy syndrome developing after cardiac surgery and its complications - tamponade and constrictive pericarditis. 


\section{Constrictive pericarditis after cardiac surgery}

Constrictive pericarditis develops in $2-3 \%$ of patients undergoing cardiac surgery (11). It usually develops late after surgery (3-56 months) and has manifestations of dyspnea and right-sided heart failure. It is thought that pooling of blood after surgery in pericardial sac, inflammation and development of fibrosis can cause the thickening and adhesions in pericardium.

One of the earliest series of cases with constrictive pericarditis after cardiac surgery and its treatment was described by Killian et al. (13). Their study included 45 patients with median age of 61 years, majority of them (33 patients) underwent coronary bypass surgery (CABG), among them 4 patients had history of 2 or more surgeries. Constrictive pericarditis developed during median 23 months (1-204 months) after surgery. Mean pericardial thickening on computed tomography was $0.8 \mathrm{~mm}$. There were signs of inflammation, fibrosis and calcification of pericardium intraoperatively. Pericardial stripping (subtotal pericardioectomy) was performed in 37 patients and 8 patients were treated medically. Overall, 28 patients had symptomatic improvement after surgery, 4 patients underwent extensive pericardioectomy. Mortality rate after surgery was $11 \%$ - 4 patients died during 30 days after pericardioectomy

Another series from Emory hospital were described by Kutcher et al. (14). Among 5207 patients undergoing cardiac surgery, 11 patients developed PPS $(0.2 \%)$ during median 82 days (4186 days) after cardiac surgery. Seven of patients had thickening of pericardium and 4 patients had both thickening and effusion. Pericardium was left open during surgery in all patients. Irrigation of pericardium with povidone-iodine solution was performed in 5 patients, with antibiotics in 2 patients and with cold saline in 4 patients. Three patients showed symptomatic relief after medical therapy, among them 1 patient underwent further removal of hematoma and 8 patients underwent radical pericardioectomy. Five patients had acute right heart overload. Six patients had STT abnormalities and 3 patients had low QRS voltage on electrocardiogram (ECG), 7 patients had paradoxal interventricular septal movement on echocardiogram and 5 patients had effusion postoperatively including 4 patients with hematoma of pericardium at posterior wall, and 10 patients had classical signs of constriction on cardiac catheterization with equalization of right ventricular, right atrial, pulmonary capillary wedge and left ventricular pressures. The standard protocol included 2-8 weeks of medical treatment with diuretic, non-steroidal anti-inflammatory agents (NSAIDs) and corticosteroids, 3 patients had symptomatic improvement and 8 patients underwent pericardioectomy. Analysis of 3 patients who had relief of symptoms on medical therapy demonstrated late cardiac tamponade or sero-effusive constriction without true fibrosis in 1 case, less advanced fibrosis with reversible seroeffusive component in $2^{\text {nd }}$ case and localized pericardial hematoma that was removed during reoperation for main indication of valvular heart disease (VHD) in 1 patient. Analysis of 8 patients who underwent pericardioectomy demonstrated extensive parietal and visceral hemorrhagic pericardial fibrosis and organization in 4 patients with inflammatory hemorrhagic fibrinous deposits. Authors concluded that almost all patients had pericardial fluid accumulation after cardiac surgery, but symptoms developed only in $15-30 \%$ of patients and despite treatment $0.2 \%$ of patients developed constrictive pericarditis.

The most recent series were reported from Cleveland clinic (15). This prospective cohort study included 239 patients with PPS after cardiac surgery (7), primary endpoints of the study were development of effusion and constriction during follow-up of 12 months. Overall 75 patients (31\%) required intervention: 44 patients with pericardial effusion and tamponade, including 22 patients who underwent pericardiocentesis and 24 - subxiphoid pericardiostomy with drainage, and 31 patients - pericardioectomy. Patients were characterized by increase of C-reactive protein and sedimentation rate, $14 \%$ had right heart failure and $31 \%$ had signs of constriction. Multivariable analysis demonstrated that constriction physiology as well as younger age, and heart failure signs increased risk of PPS and constriction development during follow-up by almost 6 times, while use of colchicine therapy and NSAIDS reduced risk of tamponade and constriction by $55 \%$ (HR - 0.45, 95\% CI 0.26 0.79 ). In younger patients with PPS with signs of heart failure and constrictive physiology, early appropriate management seems to be warranted. 


\section{Predictors and outcomes of PPS}

Several studies described predictors of PPS development after heart surgery. Analysis of COPPS prospective randomized double blind trial (1) on efficacy of colchicine after cardiac surgery for prevention of PPS included 360 patients randomized for placebo and colchicine. Among them 15\% developed PPS, with $79.6 \%$ during $1^{\text {st }}$ months after cardiac surgery, $13 \%$ - 2 months and $7.4 \%-3$ months. During follow-up tamponade was observed in $1.9 \%$ of patients, readmission in $19.8 \%$, PPS relapse in $3.7 \%$ of patients. Risk of unfavorable outcomes development was 2.32 times higher in patients with renal failure and 4.31 times higher in patients with pleural incision.

FinPPS study (16) included 688 patients undergoing CABG surgery, among them PPS developed in 61 patients $(8.9 \%)$ during median 21 days (1-52 days) of follow-up. Interventions were required in 13 patients $(22 \%)$ - pleural drainage and 3 patients $(4.9 \%)$ underwent pericardiocentesis. Patients with PPS more often received blood transfusion but had less diabetes (HR 0.32 95\% CI 0.15-0.71, p=0.005) and were less treated with metformin. Recurrence developed in $38 \%$. Predictors of PPS were blood transfusion $>1$ unit (HR 1.9 95\%CI 1.1-3.2, $\mathrm{p}=0.017$ ) and renal insufficiency, while independent predictor of recurrence was increased body mass index. Patients who developed relapse of PPS also had shorter hospital stay and more pleural punctions. In this study, bleeding played major role in patients undergoing $\mathrm{CABG}$ in development of PPS, and surprisingly diabetes played protective role.

Another study (7) that included 822 patients undergoing cardiac valve surgery addressed the risk factors and primary (1-year reoperation due to tamponade) and secondary (hospital stay duration and mortality) outcomes in patients who developed PPS after cardiac surgery. Overall $14.5 \%$ of patients developed PPS. Patients with high body mass index (OR 0.94, 95\%CI 0.89-0.99) had low risk of PPS, while preoperative treatment for chronic obstructive pulmonary disease without corticosteroids was associated with 2.55 fold (95\%CI 1.25-5.2) increased risk of PPS development. Patients with PPS had high risk of re-operation for tamponade (20.9\% vs 2.5\% (OR-15.49, 95\%CI 7.44-33.58), longer in-hospital stay (13 days vs 11 days, $\mathrm{p}=0.001)$, but no difference in mortality rate $(4.2 \%$ vs.5.5 \%) as compared to group of patients without PPS.

In our series (17), we detected development of PPS in $15.5 \%$, complicated by tamponade in $10 \%$ and constrictive pericarditis in $5 \%$ of patients.

Recent systematic review on determinants of PPS demonstrated that the risk of PPS is associated with young age patients, requirement for red blood cells transfusion, low level of preoperative platelet and hemoglobin levels (18).

Thus, patients with bleeding, low level of platelets and hemoglobin, requiring red blood cells transfusion, pleural punctions, preoperative history of treatment for chronic obstructive pulmonary disease, renal failure, are more prone to develop PPS after cardiac surgery. Overall, the PPS is associated with high-risk of tamponade development; however, the prognosis is good with no difference in mortality as compared to patients without PPS.

\section{Mechanisms of PPS and its complications development after cardiac surgery}

Several mechanisms responsible for development of PPS have been described. It is thought that spilled blood, bleeding and its contact with serosal surface has an irritating effect inducing thrombosis, inflammation and fibrosis. It was observed that intraoperative bleeding is associated with development of adhesions and fibrosis leading to constrictive pericarditis (19).

One of the frequent causes of pericardial involvement is the retained blood syndrome (RBS) that develops due to obstruction of chest tubes by clot that initiates vascular endothelial growth factor inflammatory response resulting in increase of tissue exudative permeability and collection of blood in pleura and pericardium. It is observed in about 13.8$22 \%$ of patients undergoing surgery and intervention for pericardial effusion and tamponade are required in about $2.7-6.4 \%$ of patients $(20,21)$. Its subacute form is associated with development of constrictive pericarditis in $1.2-2.0 \%$ of patients (21). RBS is defined in presence of 2 of following signs: pleural effusion/ hemothorax requiring drainage, pericardial effusion requiring drainage, reexploration for washout of blood, interventions for postoperative pericardial constriction, interventions for postoperative fibrothorax. 
Operative technique plays a major role. It was shown that leaving open pericardium after cardiac surgery may be accompanied by higher rate of constrictive pericarditis, Nandi et al. (22) demonstrated that among 56 cases with constrictive pericarditis, 33 had their pericardium opened after cardiac surgery. In series of Killian et al. (13), 16 patients had their pericardium sutured by interrupted sutures, in 2 patients pericardium was left open and closed sutures were applied in 2 patients. The chemical injury can contribute to the development of fibrosis and constriction. It was observed that use of povidone-iodine irrigation was accompanied by higher rate of constrictive pericarditis (23), as well as marked postoperative effusion and inflammation. Kuthcher et al. (14) emphasized the role of trauma during surgery, almost all patients undergoing cardiac surgery had the signs of effusion, and PPS developed in cases with failed reabsorption of effusion.

Among factors contributing to the development of constrictive component, drying of pericardium and chemical exposure might destroy normal fibrinous activity of pericardial serous tissue generating basis for development of thrombosis, adhesions and reactive fibrosis and late and immediate tamponade. Earlier studies reported that cold injury during flushing of pericardium with iced saline could stimulate fibroblasts proliferation and disruption of serous continuity (23). Use of povidone-iodine solution has not been shown to have tissue toxicity in experimental studies, but along with hemorrhage, drying procedures and using cold saline can contribute to irritation of pericardium (24).

In some severe cases there is an extensive fibrotic thickening and calcification of pericardium involving epicardium (25) and coronary vein grafts (26) buried in scarred epicardium. Specific surgical techniques as cross-hatching of epicardium have been utilized to relief signs of constriction.

Role of inflammation has been confirmed in several studies that documented increase in Creactive protein level and interleukin 8 in patients with PPS $(27,28)$. In our series (17) we showed that effusion in patients undergoing VHD surgery was correlated with signs of inflammation and presence of adhesions was related to the number of re-operations.
Another factor that can contribute to the development of late tamponade is an abnormal reaction to anticoagulant therapy in patients after VHD surgery with 2-3 fold increase in INR levels (6.0-9.0) and its association with development of late tamponade after surgery even in the absence of anticoagulant therapy (29-32).

Thus mechanical (trauma) and chemical, cold pericardial injury during cardiac operations, surgical technique, and inflammation, bleeding and abnormal reaction to anticoagulation therapy are main factors underlying development of pericardial thickening, thrombosis, fibrosis, adhesions, and effusion after cardiac surgery.

\section{Treatment}

Treatment of effusive-constrictive postcardiotomy syndrome initially should include use of diuretics, NSAIDs and anti-inflammatory therapy to reduce inflammation and immune reaction, and facilitate reabsorption of effusion (3-5). Several studies reported satisfactory results of treatment of effusive-constrictive pericarditis after cardiac surgery with steroids $(3-5,14)$. It should be also noted that use of NSAIDs might be associated with high rate of side effects (9) and steroids might increase recurrence rate of effusions and complications of PPS $(7,33)$.

Pericardioectomy is the only effective treatment of constriction due to fibrosis and thickened pericardium. In some complicated cases with involvement of epicardium the specific techniques like cross-hatching or "turtle cage" are usually applied to relief epicardial constriction $(11,25$, 26).

Treatment of effusion and tamponade is mainly interventional and re-exploration for bleeding is required if tamponade develops early after surgery. One should take in account that diagnosis of tamponade signs is challenging early after surgery and the clinical signs and diagnostic imaging might mislead, because effusions are usually localized and can cause hemodynamic compromise (signs of bleeding, oliguria, raise in right atrial pressure, low cardiac output) that should not delay aggressive surgical reexploration (12). Late tamponade usually accompanied by classical signs (12) and pericardiocentesis with or without drainage is choice of treatment. 


\section{Prevention}

Few studies demonstrated no effect of steroids before or during surgery in prevention of PPS in children and adults $(34,35)$. In DECS trial, $14.5 \%$ of 4494 patients undergoing cardiac surgery with cardiopulmonary bypass developed PPS, among them $3.5 \%$ of cases were complicated by tamponade. Intraoperative administration of dexamethazone failed to prevent development of PPS. In another randomized study, prophylactic use of methylprednisolone pre-cardiopulmonary bypass revealed no difference in the incidence of PPS between groups receiving methylprednisolone and saline; however, complications of PPS in form of tamponade were significantly higher in a group of methylprednisolone (30). In a posthoc analysis of DECS trial use of dexamethasone $1 \mathrm{mg} / \mathrm{kg}$ iv versus placebo increased risk of re-thoracotomy due to tamponade by 1.84 times (95\% CI 1.3-2.61, $\mathrm{p}<0.001)(33)$.

On the other hand, Sevuk et al. (36) reported a $20 \%$ (OR $0.8, \quad 95 \%$ CI $0.25-0.91, \quad \mathrm{p}<0.026$ ) absolute reduction of PPS risk after a single intraoperative dose of $1 \mathrm{mg} / \mathrm{kg}$ of methylprednisolone in patients undergoing coronary artery bypass surgery.

It is not clear whether it is a class effect of steroids or the type of operation, with increased risk of tamponade in patients undergoing valve surgery receiving corticosteroids. It should be taken in account that abnormal reaction to anticoagulation can also contribute to development of bleeding and pericardial involvement (29-32).

Promising results have been reported for use of colchicine (8). In a COPPS double-blind randomized trial, 360 patients were allocated into 2 arms- 180 patients receiving placebo and 180 patients receiving colchicine $-1 \mathrm{mg}$ twice daily $1^{\text {st }}$ day after surgery and then $0.5 \mathrm{mg}$ twice daily 30 days after surgery in patients $>70 \mathrm{~kg}$ and half dose for patients $<70 \mathrm{~kg}$. Follow-up was 12 months. Primary endpoint was PPS, and secondary composite endpoint included rehospitalization, constrictive pericarditis, tamponade and relapse of pericardial disease. Patients in colchicine arm developed less PPS (8.9\% vs $21.1 \%, \mathrm{p}=0.002)$ and had significantly fewer secondary composite endpoint $(0.6 \%$ vs $5.9 \%, \mathrm{p}=0.024)$. Side effects occurrence did not differ between both arms. Patients on colchicine therapy had significantly better survival free of PPS as compared to placebo group. Colchicine was found to be effective in prevention of PPS and its complications in patients undergoing cardiac surgery.

Recent meta-analysis (37) of 4 randomized controlled trials (894 pts) on prevention of PPS including $2-$ colchicine studies, 1methylprendisolone and 1 aspirin demonstrated (Fig. 1) that colchicine (2 studies $227 \mathrm{pts}$ ) reduced development of PPS by $62 \%$ (OR- $0.38,95 \%$ CI 0.22-0.65), while no effect was found for methylprednisolone or aspirin.

ESC/ EACTS recommends use of colchicine after cardiac surgery for prevention of PPS in weightadjusted doses (i.e. $0.5 \mathrm{mg}$ once for patients $\leq 70$ $\mathrm{kg}$ and $0.5 \mathrm{mg}$ twice daily for patients $70 \mathrm{~kg}$ ) and without a loading dose if there are no contraindications and it is tolerated with duration of preventive treatment for 1 month (9).

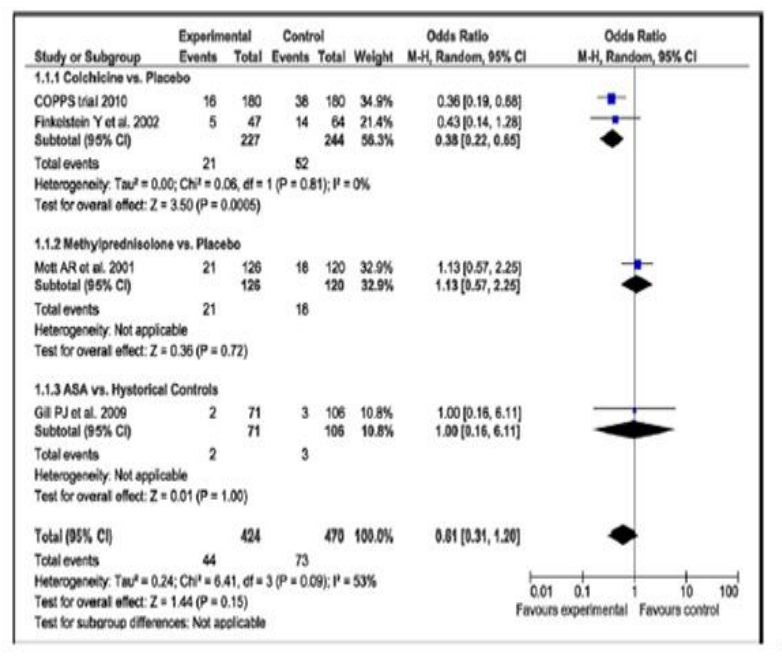

Figure 1. Forest plot for risk of postpericardiotomy syndrome summarizes and compares postpericardiotomy syndrome outcome in groups of with and without preventive treatment.

(Reproduced with permission of Elsevier Copyright 2011 from Imazio M, Brucato A, Markel G, Cemin R, Trinchero R, Spodick DH, Adler Y. Meta-Analysis of Randomized Trials Focusing on Prevention of the Postpericardiotomy Syndrome. Am J Cardiol 2011; 108: 575-9.) 


\section{Directions for future research}

Overall, there is a need for studies with a larger number of patients on intraoperative and clinical predictors and outcomes of postpericardiotomy syndrome with and without constriction and ways of prevention of its complications.

\section{Conclusion}

Postpericardiotomy syndrome develops in about $1 / 3$ of patients undergoing cardiac surgery. Though causes are not yet well-defined operative technique, pericardial injury and inflammation may play role. The prognosis when timely treated is usually favorable, however complications like tamponade and constriction may occur. If patient develops classic signs of PPS with effusion early after surgery, the recommended treatment includes treatment with nonsteroidal anti-inflammatory agents, steroids and diuretics. In case of large effusion and tamponade pericardiocentesis is indicated. In case of constrictive pericarditis after surgery, pericardiotomy is indicated. For prevention of PPS, colchicine is shown to be effective.

Peer-review: External and internal Conflict of interest: None to declare Authorship: T.K.

\section{References}

1. Imazio M, Brucato A, Rovere ME, Gandino A, Cemin R, Ferrua S, et al. Contemporary features, risk factors, and prognosis of the post-pericardiotomy syndrome. Am J Cardiol 2011;108: 1183-7.

2.Rice PL. Riffatre K, Montoya A. Constrictive pericarditis following cardiac surgery. Ann Thorac Surg 1981: 31: 450-3.

3.Kendall ME, Rhodes GR, Wolfe W. Cardiac constriction following aorta-to-coronary bypass surgery. J Thorac Cardiovasc Surg 1972; 64: 142-53.

4.Brown DF, Older T. Pericardial constriction as a late complication of coronary bypass surgery. J Thorac Cardiovasc Surg 1977; 74: 61-4.

5.Cohen MV, Greenberg MA. Constrictive pericarditis: early and late complication of cardiac surgery. Am J Cardiol 1979; 43: 657-61.
6.Kim BJ, Ma JS. Constrictive pericarditis after surgical closure of atrial septal defect in a child. J Korean Med Sci 1998; 13: 658-61.

7.Van Osch D, Dieleman JM, Bunge JJ, Van Dijk D, Doevendans PA, Suyker WJ, et al. Risk factors and prognosis of postpericardiotomy syndrome in patients undergoing valve surgery. J Thorac Cardiovasc Surg 2017; 153: 878-85.

8. Imazio M, Trinchero R, Brucato A, Rovere ME, Gandino A, Cemin R, et al; on behalf of the COPPS Investigators. COlchicine for the Prevention of the Post-pericardiotomy Syndrome (COPPS): a multicentre, randomized, double-blind, placebocontrolled trial. Eur Heart J 2010; 31: 2749-54.

9. Adler Y, Charron P, Imazio M, Badano L, BaronEsquivias G, Bogaert J, et al. 2015 ESC Guidelines for the diagnosis and management of pericardial diseases. The Task Force for the Diagnosis and Management of Pericardial Diseases of the European Society of Cardiology (ESC) Endorsed by: The European Association for Cardio-Thoracic Surgery (EACTS). Eur Heart J 2015; 36: 2921-64.

10.Patel RC, Goyal H, Shah AI, Ghali JK. Wearing the mask of ST-elevation myocardial infarction: postpericardiotomy syndrome. Am J Emerg Med 2015; 33: 1115.e5-e7

11. Gaudino M, Anselmi A, Pavone N, Massetti M. Constrictive pericarditis after cardiac surgery. Ann Thorac Surg 2013; 95: 731-6.

12. Price S, Prout J, Jaggar SI, Gibson DG, Pepper JR. 'Tamponade' following cardiac surgery: terminology and echocardiography may both mislead. Eur J Cardio-thorac Surg 2004; 26: 1156-60.

13. Killian DM, Furiasse JG, Scanlon PG, et al. Constrictive pericarditis after cardiac surgery. Am Heart J 1989:118: 563-8.

14. Kutcher MA, King SB 3rd, Alimurung BN, Craver JM, Logue RB. Constrictive pericarditis as a complication of cardiac surgery: recognition of an entity. Am J Cardiol 1992: 50: 742-8.

15. Alraies MC, Al Jaroudi W, Shabrang C, Klein AL, Tamarappoo BK. Clinical features associated with adverse events in patients with postpericardiotomy syndrome following cardiac surgery. Am J Cardiol 2014; 114: 1426-30. 
16.Lehto J, Gunn J, Karjalainen P, Airaksinen J, Kivienemi $T$. Incidence and risk factors of postpericardiotomy syndrome requiring medical attention: The Finland postpericardiotomy syndrome study. J Thorac Cardiovasc Surg 2015; 149: 1324-9.

17.Kudaiberdiev T. Clinical significance of postperiocardotomy pericardial involvement in reoperative cardiac surgery. Eur Heart J Cardiovasc Imaging Abstracts Supplement 2014: 15 (Supplement 2): ii58.

18. Van Osch D, Nathoe HM, Jacob KA, Doevendance PA, van Dijk D, Suyker WJ, et al. Determinanats of postpericardiotomy syndrome: a systematic review. Eur J Clin Investig 2017; Apr 20. Published ahead of print.

19. Ribeiro P, Sapsford R, Evans T, Parcharidis G, Oakley C. Constrictive pericarditic as a complication of coronary artery bypass surgery. Br Heart J 1984; 51: 205-10.

20. Sirch J, Ledwon M, Puski T, Boyle EM, Pfeeiffer $S$, Fischlein T. Active clearance of chest drainage catheters reduces retained blood. J Thorac Cardiovasc Surg 2016; 151: 832-8.e1-2.

21 Boyel EM, Gillinov AM, Cohn WE. Retained blood syndrome after cardiac surgery: A new look at an old problem. Innovations (Phila) 2015; 10: 296303.

22.Nandi P, Leung JS, Cheung KL. Closure of pericardium after open heart surgery. A way to prevent postoperative cardiac tamponade. Br Heart J 1976; 38: 1319-23.

23. Speicher CE, Ferrigan L, Wolfson SK Jr, Yalav $\mathrm{EH}$, Rawson AJ. Cold injury of myocardium and pericardium in cardiac hypothermia. Surg Gynecol Obstet 1962; 114: 659-65.

24.Marsa R, Mehta S, Willis W, Bailey L. Constrictive pericarditis after myocardial revascularization: report of three cases. Am J Cardiol. 1979; 44: 177-83.

25.Anderson CA, Rodriguez E, Shammas RL, Kypson AP. Early constrictive epicarditis after coronary artery bypass surgery. Ann Thorac Surg 2009; 87:642-3.

26.Bewtra C, Schultz RD. Constrictive calcific pericarditis following coronary arterial bypass surgery. Human Pathol 1985; 16: 522-5.

27. Snefjela N, Lappegard KT. Development of postpericardiotomy syndrome is preceded by an increase in pro-inflammatory and a decrease in anti- inflammatory serological markers. J Cardiothorac Surg 2012; 7: 72 .

28.Jaworska-Wilczynska M, Magalska A, Piwocka K, Szymanski P, Kusmierczyk M, Wąsik M, et al. Low interleukin - 8 level predicts the occurrence of the postpericardiotomy syndrome. Plos One 2014; 9: e108-122.

29.Wong PS, Pugsley WB. Raised international normalized ratio (INR): Is it a cause or an effect of late cardiac tamponade? Br Heart J 1992; 68: 212-3.

'30.Pepi M, Muratori M, Barbier P, Doria E, Arena V, Berti M., et al. Pericardial effusion after cardiac surgery: incidence, site, size and hemodynamic consequencies. Br Heart J 1994; 72: 327-31.

31.Malouf JF, Adam S, Charzeddine W, Stefadouros MA. The role of anticoagulation in the development of pericardial effusion and late tamponade after cardiac surgery. Eur Heart J 1993; 14: 1451-7.

32.King TE, Stelzner TJ, Sahn SA. Cardiac tamponade complicating the postpericardiotomy syndrome. Chest 1983; 83: 500-3.

33. Van Osch D, Dieleman JM, Nathoe HM, Boasson MP, Kluin J, Bunge JJ, et al. Intraoperative high-dose dexamethasone and the risk of re-thoracotomy. Ann Thorac Surg 2015; 100: 2237-42.

34.Mott AR, Fraser CD, Kusnoor AV, Giesecke NM, Reul GJ Jr, Drescher KL, et al. The effect of shortterm prophylactic methylprednisolone on the incidence and severity of postpericardiotomy syndrome in children undergoing cardiac surgery with cardiopulmonary bypass. J Am Coll Cardiol 2001; 37: 1700-6.

35.Bunge JJH, van Osch D, Dieleman JM, Jacob KA, Kluin J, van Dijk D, et al; for the Dexamethasone for Cardiac Surgery (DECS) Study Group. Dexamethasone for the prevention of postpericardiotomy syndrome: A DExamethasone for Cardiac Surgery substudy. Am Heart J 2014; 168: 126-131.e1

36. Sevuk U, Baysal E, Altindag R, Yaylak B, Adiyaman MS, Ay N, et al. Role of methylprednisolone in the prevention of postpericardiotomy syndrome after cardiac surgery. Eur Rev Med Pharmacol Sci 2016; 20: 514-9.

37.Imazio M, Brucato A, Markel G, Cemin R, Trinchero R, Spodick DH, et al. Meta-analysis of randomized trials focusing on prevention of the postpericardiotomy syndrome. Am J Cardiol 2011; 108: 575-9. 